$\mathrm{J}$ o u r n a l of

Mathematics

and Applications

JMA No 41, pp 157-170 (2018)

\title{
FG-coupled Fixed Point Theorems for Contractive Type Mappings in Partially Ordered Metric Spaces
}

\author{
Deepa Karichery and Shaini Pulickakunnel
}

\begin{abstract}
In this paper we prove FG-coupled fixed point theorems for Kannan, Reich and Chatterjea type mappings in partially ordered complete metric spaces using mixed monotone property.
\end{abstract}

AMS Subject Classification: 47H10, 54F05.

Keywords and Phrases: FG-coupled fixed point; Mixed monotone property; Contractive type mappings; Partially ordered space.

\section{Introduction and Preliminaries}

Banach contraction theorem is one of the fundamental theorems in metric fixed point theory. Banach proved existence of unique fixed point for a self contraction in complete metric space. Since the contractions are always continuous, Kannan introduced a new type of contractive map known as Kannan mapping [8] and proved analogues results of Banach contraction theorem. The importance of Kannan mapping is that it can be discontinuous and it characterizes completeness of the space $[14,15]$. In [11] Reich introduced a new type of contraction which is a generalization of Banach contraction and Kannan mapping and proved existence of unique fixed point in complete metric spaces. Later Chatterjea defined a contraction similar to Kannan mapping known as Chatterjea mapping [4] and proved various fixed point results. Inspired by these contractions, several authors did research in this area using different spaces and by weakening the contraction conditions $[2,7,9,12]$.

The concept of coupled fixed point was introduced by Guo and Lakshmikantham [6]. They proved fixed point theorems using mixed monotone property in cone spaces.

COPYRIGHT (C) by Publishing House of Rzeszów University of Technology P.O. Box 85, 35-959 Rzeszów, Poland 
In [3] Gnana Bhaskar and Lakshmikantham proved coupled fixed point theorems for contractions in partially ordered complete metric spaces using mixed monotone property. Kannan, Chatterjea and Reich type contractions are further explored in coupled fixed point theory and the results are reported in $[1,5,13]$. Recently the concept of FG-coupled fixed point was introduced in [10] and they proved FG-coupled fixed point theorems for various contractive type mappings.

In this paper we prove existence of FG-coupled fixed point theorems using Kannan, Chatterjea and Reich type contraction on partially ordered complete metric spaces.

Now we recall some basic concepts of coupled and FG-coupled fixed points.

Definition $1.1([3])$. An element $(x, y) \in X \times X$ is said to be a coupled fixed point of the map $F: X \times X \rightarrow X$ if $F(x, y)=x$ and $F(y, x)=y$.

Definition $1.2([10])$. Let $\left(X, d_{X}, \leq_{P_{1}}\right)$ and $\left(Y, d_{Y}, \leq_{P_{2}}\right)$ be two partially ordered metric spaces and $F: X \times Y \rightarrow X$ and $G: Y \times X \rightarrow Y$. We say that $F$ and $G$ have mixed monotone property if for any $x, y \in X$

$x_{1}, x_{2} \in X, \quad x_{1} \leq_{P_{1}} x_{2} \Rightarrow F\left(x_{1}, y\right) \leq_{P_{1}} F\left(x_{2}, y\right)$ and $G\left(y, x_{1}\right) \geq_{P_{2}} G\left(y, x_{2}\right)$

$y_{1}, y_{2} \in Y, \quad y_{1} \leq_{P_{2}} y_{2} \Rightarrow F\left(x, y_{1}\right) \geq_{P_{1}} F\left(x, y_{2}\right)$ and $G\left(y_{1}, x\right) \leq_{P_{2}} G\left(y_{2}, x\right)$.

Definition $1.3([10])$. An element $(x, y) \in X \times Y$ is said to be FG-coupled fixed point if $F(x, y)=x$ and $G(y, x)=y$.

If $(x, y) \in X \times Y$ is an FG-coupled fixed point then $(y, x) \in Y \times X$ is a GFcoupled fixed point. Partial order $\leq$ on $X \times Y$ is defined as $(u, v) \leq(x, y) \Leftrightarrow$ $x \geq_{P_{1}} \quad u, y \leq_{P_{2}} \quad v \forall(x, y),(u, v) \in X \times Y$. Also the iteration is given by $F^{n+1}(x, y)=F\left(F^{n}(x, y), G^{n}(y, x)\right)$ and $G^{n+1}(y, x)=G\left(G^{n}(y, x), F^{n}(x, y)\right)$ for every $n \in \mathbb{N}$ and $(x, y) \in X \times Y$.

\section{Main Results}

Theorem 2.1. Let $\left(X, d_{X}, \leq_{P_{1}}\right),\left(Y, d_{Y}, \leq_{P_{2}}\right)$ be two partially ordered complete metric spaces. Let $F: X \times Y \rightarrow X$ and $G: Y \times X \rightarrow Y$ be two continuous functions having the mixed monotone property. Assume that there exist $p, q, r, s \in\left[0, \frac{1}{2}\right)$ satisfying

$$
\begin{gathered}
d_{X}(F(x, y), F(u, v)) \leq p d_{X}(x, F(x, y))+q d_{X}(u, F(u, v)) ; \forall x \geq_{P_{1}} u, y \leq_{P_{2}} v \\
d_{Y}(G(y, x), G(v, u)) \leq r d_{Y}(y, G(y, x))+s d_{Y}(v, G(v, u)) ; \forall x \leq_{P_{1}} u, y \geq_{P_{2}} v
\end{gathered}
$$

If there exist $x_{0} \in X, y_{0} \in Y$ satisfying $x_{0} \leq_{P_{1}} F\left(x_{0}, y_{0}\right)$ and $y_{0} \geq_{P_{2}} G\left(y_{0}, x_{0}\right)$ then there exist $x \in X, y \in Y$ such that $x=F(x, y)$ and $y=G(y, x)$. 
Proof. Given $x_{0} \leq_{P_{1}} F\left(x_{0}, y_{0}\right)=x_{1}$ and $y_{0} \geq_{P_{2}} G\left(y_{0}, x_{0}\right)=y_{1}$.

Define $x_{n+1}=F\left(x_{n}, y_{n}\right)$ and $y_{n+1}=G\left(y_{n}, x_{n}\right)$ for $n=1,2,3$..

Then we can easily show that $\left\{x_{n}\right\}$ is increasing in $\mathrm{X}$ and $\left\{y_{n}\right\}$ is decreasing in $\mathrm{Y}$.

Using inequalities (1) and (2) we get

$$
\begin{aligned}
d_{X}\left(x_{n+1}, x_{n}\right) & =d_{X}\left(F\left(x_{n}, y_{n}\right), F\left(x_{n-1}, y_{n-1}\right)\right) \\
& \leq p d_{X}\left(x_{n}, F\left(x_{n}, y_{n}\right)\right)+q d_{X}\left(x_{n-1}, F\left(x_{n-1}, y_{n-1}\right)\right) \\
& =p d_{X}\left(x_{n}, x_{n+1}\right)+q d_{X}\left(x_{n-1}, x_{n}\right)
\end{aligned}
$$

ie, $(1-p) d_{X}\left(x_{n+1}, x_{n}\right) \leq q d_{X}\left(x_{n-1}, x_{n}\right)$

$$
\text { ie, } \begin{aligned}
d_{X}\left(x_{n}, x_{n+1}\right) & \leq \frac{q}{1-p} d_{X}\left(x_{n-1}, x_{n}\right) \\
& =\delta_{1} d_{X}\left(x_{n-1}, x_{n}\right) \text { where } \delta_{1}=\frac{q}{1-p}<1 \\
& \leq \delta_{1}^{2} d_{X}\left(x_{n-2}, x_{n-1}\right) \\
& \quad \vdots \\
& \leq \delta_{1}^{n} d_{X}\left(x_{0}, x_{1}\right) .
\end{aligned}
$$

Similarly we get $d_{Y}\left(y_{n+1}, y_{n}\right) \leq \delta_{2}^{n} d_{Y}\left(y_{1}, y_{0}\right)$ where $\delta_{2}=\frac{r}{1-s}<1$.

Consider $m>n$

$$
\begin{aligned}
d_{X}\left(x_{m}, x_{n}\right) & \leq d_{X}\left(x_{m}, x_{m-1}\right)+d_{X}\left(x_{m-1}, x_{m-2}\right)+\ldots+d_{X}\left(x_{n+1}, x_{n}\right) \\
& \leq \delta_{1}{ }^{m-1} d_{X}\left(x_{1}, x_{0}\right)+\delta_{1}^{m-2} d_{X}\left(x_{1}, x_{0}\right)+\ldots+\delta_{1}{ }^{n} d_{X}\left(x_{1}, x_{0}\right) \\
& =\delta_{1}{ }^{n}\left(1+\delta_{1}+\ldots+\delta_{1}^{m-n-1}\right) d_{X}\left(x_{1}, x_{0}\right) \\
& \leqslant \frac{\delta_{1}{ }^{n}}{1-\delta_{1}} d_{X}\left(x_{1}, x_{0}\right) .
\end{aligned}
$$

Since $0 \leq \delta_{1}<1, \delta_{1}{ }^{n}$ converges to 0 (as $\left.n \rightarrow \infty\right)$. Therefore $\left\{F^{n}\left(x_{0}, y_{0}\right)\right\}$ is a Cauchy sequence in $X$. Similarly we can prove that $\left\{G^{n}\left(y_{0}, x_{0}\right)\right\}$ is a Cauchy sequence in $Y$. Since by the completeness of $X$ and $Y$, there exist $x \in X$ and $y \in Y$ such that $\lim _{n \rightarrow \infty} F^{n}\left(x_{0}, y_{0}\right)=x$ and $\lim _{n \rightarrow \infty} G^{n}\left(y_{0}, x_{0}\right)=y$.

Now we have to prove the existence of FG-coupled fixed point.

Consider,

$$
\begin{aligned}
d_{X}(F(x, y), x) & =\lim _{n \rightarrow \infty} d_{X}\left(F\left(F^{n}\left(x_{0}, y_{0}\right), G^{n}\left(y_{0}, x_{0}\right)\right), F^{n}\left(x_{0}, y_{0}\right)\right) \\
& =\lim _{n \rightarrow \infty} d_{X}\left(F^{n+1}\left(x_{0}, y_{0}\right), F^{n}\left(x_{0}, y_{0}\right)\right) \\
& =0
\end{aligned}
$$

ie, $F(x, y)=x$. Similarly we get $G(y, x)=y$.

By replacing the continuity of $F$ and $G$ by other conditions we obtain the following existence theorems of FG-coupled fixed point. 
Theorem 2.2. Let $\left(X, d_{X}, \leq_{P_{1}}\right)$ and $\left(Y, d_{Y}, \leq_{P_{2}}\right)$ be two partially ordered complete metric spaces and $F: X \times Y \rightarrow X, G: Y \times X \rightarrow Y$ be two mappings having the mixed monotone property. Assume that $X$ and $Y$ satisfy the following property

(i) If a non-decreasing sequence $\left\{x_{n}\right\} \rightarrow x$ then $x_{n} \leq_{P_{1}} x \forall n$.

(ii) If a non-increasing sequence $\left\{y_{n}\right\} \rightarrow y$ then $y \leq_{P_{2}} y_{n} \forall n$.

Also assume that there exist $p, q, r, s \in\left[0, \frac{1}{2}\right)$ satisfying

$$
\begin{aligned}
& d_{X}(F(x, y), F(u, v)) \leq p d_{X}(x, F(x, y))+q d_{X}(u, F(u, v)) ; \forall x \geq_{P_{1}} u, y \leq_{P_{2}} v \\
& d_{Y}(G(y, x), G(v, u)) \leq r d_{Y}(y, G(y, x))+s d_{Y}(v, G(v, u)) ; \forall x \leq_{P_{1}} u, y \geq_{P_{2}} v .
\end{aligned}
$$

If there exist $x_{0} \in X, y_{0} \in Y$ satisfying $x_{0} \leq_{P_{1}} F\left(x_{0}, y_{0}\right)$ and $y_{0} \geq_{P_{2}} G\left(y_{0}, x_{0}\right)$ then there exist $x \in X, y \in Y$ such that $x=F(x, y)$ and $y=G(y, x)$.

Proof. Following as in the proof of Theorem 2.1 we get $\lim _{n \rightarrow \infty} F^{n}\left(x_{0}, y_{0}\right)=x$ and $\lim _{n \rightarrow \infty} G^{n}\left(y_{0}, x_{0}\right)=y$.

Now we have

$$
\begin{aligned}
d_{X}(F(x, y), x) \leq & d_{X}\left(F(x, y), F^{n+1}\left(x_{0}, y_{0}\right)\right)+d_{X}\left(F^{n+1}\left(x_{0}, y_{0}\right), x\right) \\
= & d_{X}\left(F(x, y), F\left(F^{n}\left(x_{0}, y_{0}\right), G^{n}\left(y_{0}, x_{0}\right)\right)+d_{X}\left(F^{n+1}\left(x_{0}, y_{0}\right), x\right)\right. \\
\leq & p d_{X}(x, F(x, y))+q d_{X}\left(F^{n}\left(x_{0}, y_{0}\right), F\left(F^{n}\left(x_{0}, y_{0}\right), G^{n}\left(y_{0}, x_{0}\right)\right)\right) \\
& +d_{X}\left(F^{n+1}\left(x_{0}, y_{0}\right), x\right) \quad(\operatorname{using}(3))
\end{aligned}
$$

ie, $d_{X}(F(x, y), x) \leq p d_{X}(x, F(x, y))$ as $n \rightarrow \infty$.

This holds only when $d_{X}(F(x, y), x)=0$. Therefore we get $F(x, y)=x$.

Similarly using (4) and $\lim _{n \rightarrow \infty} G^{n}\left(y_{0}, x_{0}\right)=y$ we can prove $y=G(y, x)$.

Remark 2.1. If we put $k=m$ and $l=n$ in Theorems 2.1 and 2.2, we get Theorems 2.7 and 2.8 respectively of [10].

Theorem 2.3. Let $\left(X, d_{X}, \leq_{P_{1}}\right),\left(Y, d_{Y}, \leq_{P_{2}}\right)$ be two partially ordered complete metric spaces. Let $F: X \times Y \rightarrow X$ and $G: Y \times X \rightarrow Y$ be two continuous functions having the mixed monotone property. Assume that there exist $p, q, r, s \in\left[0, \frac{1}{2}\right)$ satisfying

$$
\begin{aligned}
& d_{X}(F(x, y), F(u, v)) \leq p d_{X}(x, F(u, v))+q d_{X}(u, F(x, y)) ; \forall x \geq_{P_{1}} u, y \leq_{P_{2}} v \\
& d_{Y}(G(y, x), G(v, u)) \leq r d_{Y}(y, G(v, u))+s d_{Y}(v, G(y, x)) ; \forall x \leq_{P_{1}} u, y \geq_{P_{2}} v .
\end{aligned}
$$

If there exist $x_{0} \in X, y_{0} \in Y$ satisfying $x_{0} \leq_{P_{1}} F\left(x_{0}, y_{0}\right)$ and $y_{0} \geq_{P_{2}} G\left(y_{0}, x_{0}\right)$ then there exist $x \in X, y \in Y$ such that $x=F(x, y)$ and $y=G(y, x)$. 
Proof. As in Theorem 2.1 we have $\left\{x_{n}\right\}$ increasing in $X$ and $\left\{y_{n}\right\}$ decreasing in $Y$. We have

$$
\begin{aligned}
d_{X}\left(x_{n+1}, x_{n}\right) & =d_{X}\left(F\left(x_{n}, y_{n}\right), F\left(x_{n-1}, y_{n-1}\right)\right) \\
& \leq p d_{X}\left(x_{n}, F\left(x_{n-1}, y_{n-1}\right)\right)+q d_{X}\left(x_{n-1}, F\left(x_{n}, y_{n}\right)\right) \quad(\operatorname{Using}(5)) \\
& =p d_{X}\left(x_{n}, x_{n}\right)+q d_{X}\left(x_{n-1}, x_{n+1}\right) \\
& \leq q\left[d_{X}\left(x_{n-1}, x_{n}\right)+d_{X}\left(x_{n}, x_{n+1}\right)\right] \\
\text { ie, } d_{X}\left(x_{n}, x_{n+1}\right) \leq & \frac{q}{1-q} d_{X}\left(x_{n-1}, x_{n}\right) \\
& =\delta_{1} d_{X}\left(x_{n-1}, x_{n}\right) \quad \text { where } \delta_{1}=\frac{q}{1-q}<1 \\
\leq & \delta_{1}^{2} d_{X}\left(x_{n-2}, x_{n-1}\right) \\
& \quad \vdots \\
\leq & \delta_{1}^{n} d_{X}\left(x_{0}, x_{1}\right) .
\end{aligned}
$$

Similarly we get $d_{Y}\left(y_{n+1}, y_{n}\right) \leq \delta_{2}{ }^{n} d_{Y}\left(y_{1}, y_{0}\right)$ where $\delta_{2}=\frac{r}{1-r}<1$

Now, we prove that $\left\{F^{n}\left(x_{0}, y_{0}\right)\right\}$ and $\left\{G^{n}\left(y_{0}, x_{0}\right)\right\}$ are Cauchy sequences in $X$ and $Y$ respectively.

For $m>n$,

$$
\begin{aligned}
d_{X}\left(x_{m}, x_{n}\right) & \leq d_{X}\left(x_{m}, x_{m-1}\right)+d_{X}\left(x_{m-1}, x_{m-2}\right)+\ldots+d_{X}\left(x_{n+1}, x_{n}\right) \\
& \leq \delta_{1}{ }^{m-1} d_{X}\left(x_{1}, x_{0}\right)+\delta_{1}{ }^{m-2} d_{X}\left(x_{1}, x_{0}\right)+\ldots+\delta_{1}{ }^{n} d_{X}\left(x_{1}, x_{0}\right) \\
& \leq \frac{\delta_{1}{ }^{n}}{1-\delta_{1}} d_{X}\left(x_{1}, x_{0}\right) .
\end{aligned}
$$

Since $0 \leq \delta_{1}<1, \delta_{1}{ }^{n}$ converges to 0 (as $\left.n \rightarrow \infty\right)$. Therefore $\left\{F^{n}\left(x_{0}, y_{0}\right)\right\}$ is a Cauchy sequence in $X$.

Similarly we can prove that $\left\{G^{n}\left(y_{0}, x_{0}\right)\right\}$ is a Cauchy sequence in $Y$.

By the completeness of $X$ and $Y$, there exist $x \in X$ and $y \in Y$ such that $\lim _{n \rightarrow \infty} F^{n}\left(x_{0}, y_{0}\right)=x$ and $\lim _{n \rightarrow \infty} G^{n}\left(y_{0}, x_{0}\right)=y$.

As in the proof of Theorem 2.1 we can show that $x=F(x, y)$ and $y=G(y, x)$.

Theorem 2.4. Let $\left(X, d_{X}, \leq_{P_{1}}\right)$ and $\left(Y, d_{Y}, \leq_{P_{2}}\right)$ be two partially ordered complete metric spaces and $F: X \times Y \rightarrow X, G: Y \times X \rightarrow Y$ be two mappings having the mixed monotone property. Assume that $X$ and $Y$ satisfy the following property

(i) If a non-decreasing sequence $\left\{x_{n}\right\} \rightarrow x$ then $x_{n} \leq_{P_{1}} x \forall n$.

(ii) If a non-increasing sequence $\left\{y_{n}\right\} \rightarrow y$ then $y \leq_{P_{2}} y_{n} \forall n$.

Also assume that there exist $p, q, r, s \in\left[0, \frac{1}{2}\right)$ satisfying

$$
d_{X}(F(x, y), F(u, v)) \leq p d_{X}(x, F(u, v))+q d_{X}(u, F(x, y)) ; \forall x \geq_{P_{1}} u, y \leq_{P_{2}} v
$$




$$
d_{Y}(G(y, x), G(v, u)) \leq r d_{Y}(y, G(v, u))+s d_{Y}(v, G(y, x)) ; \forall x \leq_{P_{1}} u, y \geq_{P_{2}} v .
$$

If there exist $x_{0} \in X, y_{0} \in Y$ satisfying $x_{0} \leq_{P_{1}} F\left(x_{0}, y_{0}\right)$ and $y_{0} \geq_{P_{2}} G\left(y_{0}, x_{0}\right)$ then there exist $x \in X, y \in Y$ such that $x=F(x, y)$ and $y=G(y, x)$.

Proof. Following as in the proof of Theorem 2.3 we get $\lim _{n \rightarrow \infty} F^{n}\left(x_{0}, y_{0}\right)=x$ and $\lim _{n \rightarrow \infty} G^{n}\left(y_{0}, x_{0}\right)=y$.

Consider

$$
\begin{aligned}
d_{X}(F(x, y), x) \leq & d_{X}\left(F(x, y), F^{n+1}\left(x_{0}, y_{0}\right)\right)+d_{X}\left(F^{n+1}\left(x_{0}, y_{0}\right), x\right) \\
= & d_{X}\left(F(x, y), F\left(F^{n}\left(x_{0}, y_{0}\right), G^{n}\left(y_{0}, x_{0}\right)\right)\right)+d_{X}\left(F^{n+1}\left(x_{0}, y_{0}\right), x\right) \\
\leq & p d_{X}\left(x, F\left(\left(F^{n}\left(x_{0}, y_{0}\right), G^{n}\left(y_{0}, x_{0}\right)\right)\right)+q d_{X}\left(F^{n}\left(x_{0}, y_{0}\right), F(x, y)\right)\right. \\
& +d_{X}\left(F^{n+1}\left(x_{0}, y_{0}\right), x\right) \\
= & p d_{X}\left(x, F^{n+1}\left(x_{0}, y_{0}\right)\right)+q d_{X}\left(F^{n}\left(x_{0}, y_{0}\right), F(x, y)\right) \\
& +d_{X}\left(F^{n+1}\left(x_{0}, y_{0}\right), x\right)
\end{aligned}
$$

ie, $d_{X}(F(x, y), x) \leq q d_{X}(x, F(x, y))$ as $n \rightarrow \infty$, which implies that $d_{X}(F(x, y), x)=0$. Therefore we get $F(x, y)=x$.

Similarly using (8) and $\lim _{n \rightarrow \infty} G^{n}\left(y_{0}, x_{0}\right)=y$, we get $y=G(y, x)$.

Remark 2.2. If we put $p=r$ and $q=s$ in Theorems 2.3 and 2.4, we get Theorems 2.9 and 2.10 respectively of [10].

The following example illustrates the above results.

Example 2.1. Let $X=[0,1]$ and $Y=[-1,1]$ with usual metric. Partial order on $X$ is defined as $x \leq_{P_{1}} u$ if and only if $x=u$ and partial order on $Y$ is defined as $y \leq_{P_{2}} v$ if and only if either $y=v$ or $(y, v)=(0,1)$. The mapping $F: X \times Y \rightarrow X$ is defined by $F(x, y)=\frac{x+1}{2}$ and $G: Y \times X \rightarrow Y$ is defined as $G(y, x)=\frac{x-1}{2}$. Then $F$ and $G$ satisfies (1), (2), (5), (6) with $p, q, r, s \in\left[0, \frac{1}{2}\right)$. Also $(1,0)$ is the FG-coupled fixed point.

Theorem 2.5. Let $\left(X, d_{X}, \leq_{P_{1}}\right),\left(Y, d_{Y}, \leq_{P_{2}}\right)$ be two partially ordered complete metric spaces. Let $F: X \times Y \rightarrow X$ and $G: Y \times X \rightarrow Y$ be two continuous functions having the mixed monotone property. Assume that there exist $a, b, c$ with $a+b+c<1$ satisfying

$$
\begin{array}{r}
d_{X}(F(x, y), F(u, v)) \leq a d_{X}(x, F(x, y))+b d_{X}(u, F(u, v))+c d_{X}(x, u) \\
\forall x \geq_{P_{1}} u, y \leq_{P_{2}} v \\
d_{Y}(G(y, x), G(v, u)) \leq a d_{Y}(y, G(y, x))+b d_{Y}(v, G(v, u))+c d_{Y}(y, v) \\
\forall x \leq_{P_{1}} u, y \geq_{P_{2}} v .
\end{array}
$$

If there exist $x_{0} \in X, y_{0} \in Y$ satisfying $x_{0} \leq_{P_{1}} F\left(x_{0}, y_{0}\right)$ and $y_{0} \geq_{P_{2}} G\left(y_{0}, x_{0}\right)$ then there exist $x \in X, y \in Y$ such that $x=F(x, y)$ and $y=G(y, x)$. 
Proof. Following as in Theorem 2.1 we have $\left\{x_{n}\right\}$ is increasing in $X$ and $\left\{y_{n}\right\}$ is decreasing in $Y$.

Now we claim that

$$
\begin{aligned}
& d_{X}\left(F^{n+1}\left(x_{0}, y_{0}\right), F^{n}\left(x_{0}, y_{0}\right)\right) \leq\left(\frac{b+c}{1-a}\right)^{n} d_{X}\left(x_{0}, x_{1}\right) \\
& d_{Y}\left(G^{n+1}\left(y_{0}, x_{0}\right), G^{n}\left(y_{0}, x_{0}\right)\right) \leq\left(\frac{a+c}{1-b}\right)^{n} d_{Y}\left(y_{0}, y_{1}\right) .
\end{aligned}
$$

The proof is by mathematical induction with the help of (9) and (10). For $n=1$, consider

$$
\begin{aligned}
d_{X}\left(F^{2}\left(x_{0}, y_{0}\right), F\left(x_{0}, y_{0}\right)\right)= & d_{X}\left(F\left(F\left(x_{0}, y_{0}\right), G\left(y_{0}, x_{0}\right)\right), F\left(x_{0}, y_{0}\right)\right) \\
\leq & a d_{X}\left(F\left(x_{0}, y_{0}\right), F^{2}\left(x_{0}, y_{0}\right)\right)+b d_{X}\left(x_{0}, F\left(x_{0}, y_{0}\right)\right) \\
& +c d_{X}\left(F\left(x_{0}, y_{0}\right), x_{0}\right) \\
\text { ie, } d_{X}\left(F^{2}\left(x_{0}, y_{0}\right), F\left(x_{0}, y_{0}\right)\right) \leq & \frac{b+c}{1-a} d_{X}\left(x_{0}, x_{1}\right) .
\end{aligned}
$$

Thus the inequality (11) is true for $n=1$.

Now assume that (11) is true for $n \leq m$, and check for $n=m+1$.

Consider,

$$
\begin{aligned}
& d_{X}\left(F^{m+2}\left(x_{0}, y_{0}\right), F^{m+1}\left(x_{0}, y_{0}\right)\right) \\
& \quad=d_{X}\left(F\left(F^{m+1}\left(x_{0}, y_{0}\right), G^{m+1}\left(y_{0}, x_{0}\right)\right), F\left(F^{m}\left(x_{0}, y_{0}\right), G^{m}\left(y_{0}, x_{0}\right)\right)\right) \\
& \quad \leq a d_{X}\left(F^{m+1}\left(x_{0}, y_{0}\right), F^{m+2}\left(x_{0}, y_{0}\right)\right)+b d_{X}\left(F^{m}\left(x_{0}, y_{0}\right), F^{m+1}\left(x_{0}, y_{0}\right)\right) \\
& \quad+c d_{X}\left(F^{m+1}\left(x_{0}, y_{0}\right), F^{m}\left(x_{0}, y_{0}\right)\right) \\
& \text { ie, } \begin{aligned}
d_{X}\left(F^{m+2}\left(x_{0}, y_{0}\right), F^{m+1}\left(x_{0}, y_{0}\right)\right) & \leq \frac{b+c}{1-a} d_{X}\left(F^{m}\left(x_{0}, y_{0}\right), F^{m+1}\left(x_{0}, y_{0}\right)\right) \\
& \leq\left(\frac{b+c}{1-a}\right)^{m+1} d_{X}\left(x_{0}, x_{1}\right)
\end{aligned}
\end{aligned}
$$

ie, the inequality (11) is true for all $n \in \mathbb{N}$.

Similarly we can prove the inequality (12).

For $m>n$, consider

$$
\begin{aligned}
d_{X}( & \left.F^{n}\left(x_{0}, y_{0}\right), F^{m}\left(x_{0}, y_{0}\right)\right) \\
& \leq d_{X}\left(F^{n}\left(x_{0}, y_{0}\right), F^{n+1}\left(x_{0}, y_{0}\right)\right)+d_{X}\left(F^{n+1}\left(x_{0}, y_{0}\right), F^{n+2}\left(x_{0}, y_{0}\right)\right)+\ldots \\
& +d_{X}\left(F^{m-1}\left(x_{0}, y_{0}\right), F^{m}\left(x_{0}, y_{0}\right)\right) \\
& \leq\left[\left(\frac{b+c}{1-a}\right)^{n}+\left(\frac{b+c}{1-a}\right)^{n+1}+\ldots+\left(\frac{b+c}{1-a}\right)^{m-1}\right] d_{X}\left(x_{0}, x_{1}\right) \\
& \leq \frac{\delta_{1}^{n}}{1-\delta_{1}} d_{X}\left(x_{0}, x_{1}\right) \text { where } \delta_{1}=\frac{b+c}{1-a}<1
\end{aligned}
$$


Since $0 \leq \delta_{1}<1, \delta_{1}{ }^{n}$ converges to 0 (as $n \rightarrow \infty$ ) ie, $\left\{F^{n}\left(x_{0}, y_{0}\right)\right\}$ is a Cauchy sequence in X. Similarly by using inequality (12) we can prove that $\left\{G^{n}\left(y_{0}, x_{0}\right)\right\}$ is a Cauchy sequence in $\mathrm{Y}$.

By the completeness of $\mathrm{X}$ and $\mathrm{Y}$, there exist $x \in X$ and $y \in Y$ such that $\lim _{n \rightarrow \infty} F^{n}\left(x_{0}, y_{0}\right)=x$ and $\lim _{n \rightarrow \infty} G^{n}\left(y_{0}, x_{0}\right)=y$.

As in the proof of Theorem 2.1, using continuity of $F$ and $G$ we can prove that $F(x, y)=x$ and $G(y, x)=y$.

If we take $X=Y$ and $F=G$ in the above theorem we get the following corollary.

Corollary 2.1. Let $(X, d, \leq)$ be a partially ordered complete metric space. Let $F: X \times X \rightarrow X$ be a continuous function having the mixed monotone property. Assume that there exist non-negative $a, b, c$ such that $a+b+c<1$ satisfying

$$
d(F(x, y), F(u, v)) \leq a d(x, F(x, y))+b d(u, F(u, v))+c d(x, u) ; \forall x \geq u, y \leq v .
$$

If there exist $x_{0}, y_{0} \in X$ satisfying $x_{0} \leq F\left(x_{0}, y_{0}\right)$ and $y_{0} \geq F\left(y_{0}, x_{0}\right)$ then there exist $(x, y) \in X \times X$ such that $x=F(x, y)$ and $y=F(y, x)$.

Theorem 2.6. Let $\left(X, d_{X}, \leq_{P_{1}}\right)$ and $\left(Y, d_{Y}, \leq_{P_{2}}\right)$ be two partially ordered complete metric spaces and $F: X \times Y \rightarrow X, G: Y \times X \rightarrow Y$ be two mappings having the mixed monotone property. Assume that $X$ and $Y$ satisfy the following property

(i) If a non-decreasing sequence $\left\{x_{n}\right\} \rightarrow x$ then $x_{n} \leq_{P_{1}} x \forall n$.

(ii) If a non-increasing sequence $\left\{y_{n}\right\} \rightarrow y$ then $y \leq_{P_{2}} y_{n} \forall n$.

Also asuume that there exist $a, b, c$ with $a+b+c<1$ satisfying

$$
\begin{array}{r}
d_{X}(F(x, y), F(u, v)) \leq a d_{X}(x, F(x, y))+b d_{X}(u, F(u, v))+c d_{X}(x, u) ; \\
\forall x \geq_{p_{1}} u, y \leq_{p_{2}} v \\
d_{Y}(G(y, x), G(v, u)) \leq a d_{Y}(y, G(y, x))+b d_{Y}(v, G(v, u))+c d_{Y}(y, v) ; \\
\forall x \leq_{P_{1}} u, y \geq_{P_{2}} v .
\end{array}
$$

If there exist $x_{0} \in X, y_{0} \in Y$ satisfying $x_{0} \leq_{P_{1}} F\left(x_{0}, y_{0}\right)$ and $y_{0} \geq_{P_{2}} G\left(y_{0}, x_{0}\right)$ then there exist $x \in X, y \in Y$ such that $x=F(x, y)$ and $y=G(y, x)$.

Proof. Following as in the proof of Theorem 2.5 we obtain $\lim _{n \rightarrow \infty} F^{n}\left(x_{0}, y_{0}\right)=x$ and $\lim _{n \rightarrow \infty} G^{n}\left(y_{0}, x_{0}\right)=y$.

We have

$$
\begin{aligned}
d_{X}(F(x, y), x) \leq & d_{X}\left(F(x, y), F^{n+1}\left(x_{0}, y_{0}\right)\right)+d_{X}\left(F^{n+1}\left(x_{0}, y_{0}\right), x\right) \\
= & d_{X}\left(F(x, y), F\left(F^{n}\left(x_{0}, y_{0}\right), G^{n}\left(y_{0}, x_{0}\right)\right)\right)+d_{X}\left(F^{n+1}\left(x_{0}, y_{0}\right), x\right) \\
\leq & a d_{X}(x, F(x, y))+b d_{X}\left(F^{n}\left(x_{0}, y_{0}\right), F\left(F^{n}\left(x_{0}, y_{0}\right), G^{n}\left(y_{0}, x_{0}\right)\right)\right) \\
& +c d_{X}\left(x, F^{n}\left(x_{0}, y_{0}\right)\right)+d_{X}\left(F^{n+1}\left(x_{0}, y_{0}\right), x\right) \\
= & a d_{X}(x, F(x, y))+b d_{X}\left(F^{n}\left(x_{0}, y_{0}\right), F^{n+1}\left(x_{0}, y_{0}\right)\right) \\
& +c d_{X}\left(x, F^{n}\left(x_{0}, y_{0}\right)\right)+d_{X}\left(F^{n+1}\left(x_{0}, y_{0}\right), x\right)
\end{aligned}
$$


ie, $d_{X}(F(x, y), x) \leq a d_{X}(x, F(x, y))$ as $n \rightarrow \infty$, which implies that $d_{X}(F(x, y), x)=0$. Therefore $F(x, y)=x$.

Similarly using (14) and $\lim _{n \rightarrow \infty} G^{n}\left(y_{0}, x_{0}\right)=y$ we get $y=G(y, x)$.

By assuming $X=Y$ and $F=G$ in the above theorem we will get the following corollary.

Corollary 2.2. Let $(X, d, \leq)$ be a partially ordered complete metric space and $F: X \times X \rightarrow X$ be a mapping having the mixed monotone property. Assume that $X$ satisfy the following property

(i) If a non-decreasing sequence $\left\{x_{n}\right\} \rightarrow x$ then $x_{n} \leq x \forall n$.

(ii) If a non-increasing sequence $\left\{y_{n}\right\} \rightarrow y$ then $y \leq y_{n} \forall n$.

Also assume that there exist non-negative $a, b, c$ such that $a+b+c<1$ satisfying

$$
d(F(x, y), F(u, v)) \leq a d(x, F(x, y))+b d(u, F(u, v))+c d(x, u) ; \forall x \geq u, y \leq v .
$$

If there exist $\left(x_{0}, y_{0}\right) \in X \times X$ satisfying $x_{0} \leq F\left(x_{0}, y_{0}\right)$ and $y_{0} \geq F\left(y_{0}, x_{0}\right)$ then there exist $x, y \in X$ such that $x=F(x, y)$ and $y=F(y, x)$.

Remark 2.3. If we take $c=0$ in Theorems 2.5 and 2.6, we get Theorems 2.7 and 2.8 respectively of $[10]$.

Theorem 2.7. Let $\left(X, d_{X}, \leq_{P_{1}}\right),\left(Y, d_{Y}, \leq_{P_{2}}\right)$ be two partially ordered complete metric spaces. Let $F: X \times Y \rightarrow X$ and $G: Y \times X \rightarrow Y$ be two continuous functions having the mixed monotone property. Assume that there exist non-negative a,b,c satisfying

$$
\begin{array}{r}
d_{X}(F(x, y), F(u, v)) \leq a d_{X}(x, F(u, v))+b d_{X}(u, F(x, y))+c d_{X}(x, u) \\
\forall x \geq_{P_{1}} u, y \leq_{P_{2}} v ; 2 b+c<1 \\
d_{Y}(G(y, x), G(v, u)) \leq a d_{Y}(y, G(v, u))+b d_{Y}(v, G(y, x))+c d_{Y}(y, v) \\
\forall x \leq_{P_{1}} u, y \geq_{P 2} v ; 2 a+c<1 .
\end{array}
$$

If there exist $x_{0} \in X, y_{0} \in Y$ satisfying $x_{0} \leq_{P_{1}} F\left(x_{0}, y_{0}\right)$ and $y_{0} \geq_{P_{2}} G\left(y_{0}, x_{0}\right)$ then there exist $x \in X, y \in Y$ such that $x=F(x, y)$ and $y=G(y, x)$.

Proof. As in the proof of Theorem 2.1, it can be proved that $\left\{x_{n}\right\}$ is increasing in $\mathrm{X}$ and $\left\{y_{n}\right\}$ is decreasing in $\mathrm{Y}$.

Now we claim that

$$
\begin{aligned}
& d_{X}\left(F^{n+1}\left(x_{0}, y_{0}\right), F^{n}\left(x_{0}, y_{0}\right)\right) \leq\left(\frac{b+c}{1-b}\right)^{n} d_{X}\left(x_{0}, x_{1}\right) \\
& d_{Y}\left(G^{n+1}\left(y_{0}, x_{0}\right), G^{n}\left(y_{0}, x_{0}\right)\right) \leq\left(\frac{a+c}{1-a}\right)^{n} d_{Y}\left(y_{0}, y_{1}\right) .
\end{aligned}
$$


We prove the claim by mathematical induction, using (15) and (16).

For $n=1$, consider

$$
\begin{aligned}
d_{X}\left(F ^ { 2 } \left(x_{0},\right.\right. & \left.\left.y_{0}\right), F\left(x_{0}, y_{0}\right)\right) \\
& =d_{X}\left(F\left(F\left(x_{0}, y_{0}\right), G\left(y_{0}, x_{0}\right)\right), F\left(x_{0}, y_{0}\right)\right) \\
& \leq a d_{X}\left(F\left(x_{0}, y_{0}\right), F\left(x_{0}, y_{0}\right)\right)+b d_{X}\left(x_{0}, F^{2}\left(x_{0}, y_{0}\right)\right)+c d_{X}\left(F\left(x_{0}, y_{0}\right), x_{0}\right) \\
& \leq b\left[d_{X}\left(x_{0}, F\left(x_{0}, y_{0}\right)\right)+d_{X}\left(F\left(x_{0}, y_{0}\right), F^{2}\left(x_{0}, y_{0}\right)\right)+c d_{X}\left(F\left(x_{0}, y_{0}\right), x_{0}\right)\right.
\end{aligned}
$$

ie, $d_{X}\left(F^{2}\left(x_{0}, y_{0}\right), F\left(x_{0}, y_{0}\right)\right) \leq \frac{b+c}{1-b} d_{X}\left(x_{0}, x_{1}\right)$.

Thus the inequality (17) is true for $n=1$.

Now assume that (17) is true for $n \leq m$, then check for $n=m+1$.

Consider,

$$
\begin{aligned}
d_{X}\left(F^{m+2}(\right. & \left.\left.x_{0}, y_{0}\right), F^{m+1}\left(x_{0}, y_{0}\right)\right) \\
& =d_{X}\left(F\left(F^{m+1}\left(x_{0}, y_{0}\right), G^{m+1}\left(y_{0}, x_{0}\right)\right), F\left(F^{m}\left(x_{0}, y_{0}\right), G^{m}\left(y_{0}, x_{0}\right)\right)\right) \\
& \leq a d_{X}\left(F^{m+1}\left(x_{0}, y_{0}\right), F^{m+1}\left(x_{0}, y_{0}\right)\right)+b d_{X}\left(F^{m}\left(x_{0}, y_{0}\right), F^{m+2}\left(x_{0}, y_{0}\right)\right) \\
& +c d_{X}\left(F^{m+1}\left(x_{0}, y_{0}\right), F^{m}\left(x_{0}, y_{0}\right)\right) \\
& \leq b\left[d_{X}\left(F^{m}\left(x_{0}, y_{0}\right), F^{m+1}\left(x_{0}, y_{0}\right)\right)+d_{X}\left(F^{m+1}\left(x_{0}, y_{0}\right), F^{m+2}\left(x_{0}, y_{0}\right)\right)\right] \\
& +c d_{X}\left(F^{m+1}\left(x_{0}, y_{0}\right), F^{m}\left(x_{0}, y_{0}\right)\right)
\end{aligned}
$$

ie,

$$
\begin{aligned}
d_{X}\left(F^{m+2}\left(x_{0}, y_{0}\right), F^{m+1}\left(x_{0}, y_{0}\right)\right) & \leq \frac{b+c}{1-b} d_{X}\left(F^{m}\left(x_{0}, y_{0}\right), F^{m+1}\left(x_{0}, y_{0}\right)\right) \\
& \leq\left(\frac{b+c}{1-b}\right)^{m+1} d_{X}\left(x_{0}, x_{1}\right)
\end{aligned}
$$

ie, the inequality (17) is true for all $n \in \mathbb{N}$.

Similarly we can prove the inequality (18).

For $m>n$, consider

$$
\begin{aligned}
d_{X}\left(F ^ { n } \left(x_{0},\right.\right. & \left.\left.y_{0}\right), F^{m}\left(x_{0}, y_{0}\right)\right) \\
& \leq d_{X}\left(F^{n}\left(x_{0}, y_{0}\right), F^{n+1}\left(x_{0}, y_{0}\right)\right)+d_{X}\left(F^{n+1}\left(x_{0}, y_{0}\right), F^{n+2}\left(x_{0}, y_{0}\right)\right)+\ldots \\
& +d_{X}\left(F^{m-1}\left(x_{0}, y_{0}\right), F^{m}\left(x_{0}, y_{0}\right)\right) \\
& \leq\left[\left(\frac{b+c}{1-b}\right)^{n}+\left(\frac{b+c}{1-b}\right)^{n+1}+\ldots+\left(\frac{b+c}{1-b}\right)^{m-1}\right] d_{X}\left(x_{0}, x_{1}\right) \\
& \leq \frac{\delta_{1}^{n}}{1-\delta_{1}} d_{X}\left(x_{0}, x_{1}\right) ; \text { where } \delta_{1}=\frac{b+c}{1-b}<1 .
\end{aligned}
$$

Since $0 \leq \delta_{1}<1, \delta_{1}{ }^{n}$ converges to 0 (as $\left.n \rightarrow \infty\right)$ ie, $\left\{F^{n}\left(x_{0}, y_{0}\right)\right\}$ is a Cauchy sequence in $X$. Similarly we can prove that $\left\{G^{n}\left(y_{0}, x_{0}\right)\right\}$ is a Cauchy sequence in $Y$. 
Since $X$ and $Y$ are complete, there exist $x \in X$ and $y \in Y$ such that $\lim _{n \rightarrow \infty} F^{n}\left(x_{0}, y_{0}\right)$ $=x$ and $\lim _{n \rightarrow \infty} G^{n}\left(y_{0}, x_{0}\right)=y$.

By continuity of $F$ and $G$, as in the Theorem 2.1 we can show that $F(x, y)=x$ and $G(y, x)=y$.

If $X=Y$ and $F=G$ in the above theorem we get the following corollary.

Corollary 2.3. Let $(X, d, \leq)$ be a partially ordered complete metric space. Let $F: X \times X \rightarrow X$ be a continuous function having the mixed monotone property. Assume that there exist non-negative $a, b, c$ such that $2 a+c<1$ and $2 b+c<1$ satisfying

$$
d(F(x, y), F(u, v)) \leq a d(x, F(u, v))+b d(u, F(x, y))+c d(x, u) ; \forall x \geq u, y \leq v .
$$

If there exist $\left(x_{0}, y_{0}\right) \in X \times Y$ satisfying $x_{0} \leq F\left(x_{0}, y_{0}\right)$ and $y_{0} \geq F\left(y_{0}, x_{0}\right)$ then there exist $x, y \in X$ such that $x=F(x, y)$ and $y=F(y, x)$.

In the following theorem we replace the continuity by other conditions to obtain FG-coupled fixed point.

Theorem 2.8. Let $\left(X, d_{X}, \leq_{P_{1}}\right)$ and $\left(Y, d_{Y}, \leq_{P_{2}}\right)$ be two partially ordered complete metric spaces and $F: X \times Y \rightarrow X, G: Y \times X \rightarrow Y$ be two mappings having the mixed monotone property. Assume that $X$ and $Y$ satisfy the following property

(i) If a non-decreasing sequence $\left\{x_{n}\right\} \rightarrow x$ then $x_{n} \leq_{P_{1}} x \forall n$.

(ii) If a non-increasing sequence $\left\{y_{n}\right\} \rightarrow y$ then $y \leq_{P_{2}} y_{n} \forall n$.

Also assume that there exist non-negative a,b,c satisfying

$$
\begin{array}{r}
d_{X}(F(x, y), F(u, v)) \leq a d_{X}(x, F(u, v))+b d_{X}(u, F(x, y))+c d_{X}(x, u) \\
\forall x \geq_{P_{1}} u, y \leq_{P_{2}} v ; 2 b+c<1 \\
d_{Y}(G(y, x), G(v, u)) \leq a d_{Y}(y, G(v, u))+b d_{Y}(v, G(y, x))+c d_{Y}(y, v) ; \\
\forall x \leq_{P_{1}} u, y \geq_{P_{2}} v ; 2 a+c<1 .
\end{array}
$$

If there exist $x_{0} \in X, y_{0} \in Y$ satisfying $x_{0} \leq_{P_{1}} F\left(x_{0}, y_{0}\right)$ and $y_{0} \geq_{P_{2}} G\left(y_{0}, x_{0}\right)$ then there exist $x \in X, y \in Y$ such that $x=F(x, y)$ and $y=G(y, x)$.

Proof. Following as in the proof of Theorem 2.7 we get $\lim _{n \rightarrow \infty} F^{n}\left(x_{0}, y_{0}\right)=x$ and $\lim _{n \rightarrow \infty} G^{n}\left(y_{0}, x_{0}\right)=y$.

We have

$$
\begin{aligned}
d_{X}(F(x, y), x) \leq & d_{X}\left(F(x, y), F^{n+1}\left(x_{0}, y_{0}\right)\right)+d_{X}\left(F^{n+1}\left(x_{0}, y_{0}\right), x\right) \\
= & d_{X}\left(F(x, y), F\left(F^{n}\left(x_{0}, y_{0}\right), G^{n}\left(y_{0}, x_{0}\right)\right)+d_{X}\left(F^{n+1}\left(x_{0}, y_{0}\right), x\right)\right. \\
\leq & a d_{X}\left(x, F\left(F^{n}\left(x_{0}, y_{0}\right), G^{n}\left(y_{0}, x_{0}\right)\right)+b d_{X}\left(F^{n}\left(x_{0}, y_{0}\right), F(x, y)\right)\right. \\
& +c d_{X}\left(x, F^{n}\left(x_{0}, y_{0}\right)\right)+d_{X}\left(F^{n+1}\left(x_{0}, y_{0}\right), x\right) \\
= & a d_{X}\left(x, F^{n+1}\left(x_{0}, y_{0}\right)\right)+b d_{X}\left(F^{n}\left(x_{0}, y_{0}\right), F(x, y)\right) \\
& +c d_{X}\left(x, F^{n}\left(x_{0}, y_{0}\right)\right)+d_{X}\left(F^{n+1}\left(x_{0}, y_{0}\right), x\right)
\end{aligned}
$$


ie, $d_{X}(F(x, y), x) \leq b d_{X}(x, F(x, y))$ as $n \rightarrow \infty$, which implies that $d_{X}(F(x, y), x)=0$. Therefore $F(x, y)=x$.

Also by using (20) and $\lim _{n \rightarrow \infty} G^{n}\left(y_{0}, x_{0}\right)=y$ we can show that $y=G(y, x)$.

Taking $X=Y$ and $F=G$ in the above corollary we get the corresponding coupled fixed point result.

Corollary 2.4. Let $(X, d, \leq)$ be a partially ordered complete metric spaces and $F: X \times Y \rightarrow X$ be a mapping having the mixed monotone property. Assume that $X$ satisfy the following property

(i) If a non-decreasing sequence $\left\{x_{n}\right\} \rightarrow x$ then $x_{n} \leq x \forall n$.

(ii) If a non-increasing sequence $\left\{y_{n}\right\} \rightarrow y$ then $y \leq y_{n} \forall n$.

Also assume that there exist non-negative $a, b, c$ such that $2 a+c<1$ and $2 b+c<1$ satisfying

$$
d(F(x, y), F(u, v)) \leq a d(x, F(u, v))+b d(u, F(x, y))+c d(x, u) ; \forall x \geq u, y \leq v .
$$

If there exist $\left(x_{0}, y_{0}\right) \in X \times Y$ satisfying $x_{0} \leq F\left(x_{0}, y_{0}\right)$ and $y_{0} \geq F\left(y_{0}, x_{0}\right)$ then there exist $(x, y) \in X \times Y$ such that $x=F(x, y)$ and $y=F(y, x)$.

Remark 2.4. If we take $c=0$ in Theorems 2.7 and 2.8, we get Theorems 2.9 and 2.10 respectively of [10].

\section{Acknowledgment}

The first author acknowledges financial support from Kerala State Council for Science, Technology and Environment (KSCSTE), in the form of fellowship. We also acknowledge the valuable suggestions made by the referee for improving this paper.

\section{References}

[1] S.M. Alsulami, The uniqueness of coupled fixed point for weakly $C$-contractive mappings in partial metric spaces, Gulf Journal of Mathematics 2 (3) (2014) $71-80$.

[2] M. Arshad, A. Shoaib, M. Abbas, A. Azam, Fixed points of a pair of Kannan type mappings on a closed ball in ordered partial metric spaces, Miskolc Mathematical Notes 14 (3) (2013) 769-784.

[3] T. Gnana Bhaskar, V. Lakshmikantham, Fixed point theorems in partially ordered metric spaces and applications, Nonlinear Anal. 65 (7) (2006) 1379-1393.

[4] S.K. Chatterjea, Fixed point theorems, C.R. Acad. Bulgare Sci. 25 (1972) 727 730. 
[5] B.S. Choudhury, P. Maity, Cyclic coupled fixed point result using Kannan type contractions, Journal of Operators 2014 (2014), Article ID 876749, http://dx.doi.org/10.1155/2014/876749.

[6] D. Guo, V. Lakshmikantham, Coupled fixed points of non-linear operators with applications, Nonlinear Analysis, Theory, Methods and Applications 11 (5) (1987) 623-632.

[7] N. Hussain, V. Parvaneh, B. Samet, C. Vetro, Some fixed point theorems for generalized contractive mappings in complete metric spaces, Fixed Point Theory and Applications (2015), DOI: 10.1186/s13663-015-0433-z.

[8] R. Kannan, Some results on fixed points, Bull. Culcutta Math. Soc. 10 (1968) 71-76.

[9] J. Olaleru, V. Olisama, M. Abbas, Coupled best proximity points of generalised Hardy-Rogers type cyclic $(\omega)$-contraction mappings, International Journal of Mathematical Analysis and Optimization: Theory and Applications 1 (2015) 33-54.

[10] E. Prajisha, P. Shaini, FG-coupled fixed point theorems for various contractions in partially ordered metric spaces, http://arxiv.org/abs/1602.00543.

[11] S. Reich, Some remarks concerning contraction mappings, Canad. Math. Bull. 14 (1) (1971) 121-124.

[12] S. Reich, Fixed points of contractive functions, Bull. Un. Math. Ital. 4 (1972) $26-42$.

[13] F. Sabetghadam, H.P. Masiha, A.H. Sanatpour, Some coupled fixed point theorems in cone metric spaces, Fixed Point Theory and Applications 2009 (2009), Article ID 125426, DOI: 10.1155/2009/125426.

[14] N. Shioji, T. Suzuki, W. Takahashi, Contractive mappings, Kannan mappings and metric completeness, Proceedings of the American Mathematical Society 126 (10) (1998) 3117-3124.

[15] P.V. Subrahmanyam, Completeness and fixed-points, Monatshefte für Mathematik 80 (4) (1975) 325-330.

DOI: $10.7862 /$ rf.2018.11

\section{Deepa Karichery}

email: deepakarichery@gmail.com

ORCID: 0000-0002-4016-4814

Department of Mathematics 
Central University of Kerala INDIA - 671316

\section{Shaini Pulickakunnel} email: shainipv@gmail.com ORCID: 0000-0001-9958-9211 Department of Mathematics Central University of Kerala INDIA - 671316

Received 05.01.2018
Accepted 10.08.2018 